\title{
A bipartite U1 site represses U1A expression by synergizing with PIE to inhibit nuclear polyadenylation
}

\author{
FEI GUAN, ${ }^{1}$ ROSE M. CARATOZZOLO, ${ }^{1}$ RAFAL GORACZNIAK, ERIC S. HO, and SAMUEL I. GUNDERSON \\ Department of Molecular Biology and Biochemistry, Rutgers University, Piscataway, New Jersey 08854, USA
}

\begin{abstract}
U1A protein negatively autoregulates itself by polyadenylation inhibition of its own pre-mRNA by binding as two molecules to a 3'UTR-located Polyadenylation Inhibitory Element (PIE). The (U1A)2-PIE complex specifically blocks U1A mRNA biosynthesis by inhibiting polyA tail addition, leading to lower mRNA levels. U1 snRNP bound to a 5'ss-like sequence, which we call a U1 site, in the 3'UTRs of certain papillomaviruses leads to inhibition of viral late gene expression via a similar mechanism. Although such U1 sites can also be artificially used to potently silence reporter and endogenous genes, no naturally occurring U1 sites have been found in eukaryotic genes. Here we identify a conserved U1 site in the human U1A gene that is, unexpectedly, within a bipartite element where the other part represses the $\mathrm{U} 1$ site via a base-pairing mechanism. The bipartite element inhibits U1A expression via a synergistic action with the nearby PIE. Unexpectedly, synergy is not based on stabilizing binding of the inhibitory factors to the $3^{\prime}$ UTR, but rather is a property of the larger ternary complex. Inhibition targets the biosynthetic step of polyA tail addition rather than altering $m$ RNA stability. This is the first example of a functional U1 site in a cellular gene and of a single gene containing two dissimilar elements that inhibit nuclear polyadenylation. Parallels with other examples where U1 snRNP inhibits expression are discussed. We expect that other cellular genes will harbor functional U1 sites.
\end{abstract}

Keywords: pre-mRNA processing; nuclear polyadenylation; U1 snRNP; U1A

\section{INTRODUCTION}

Almost all eukaryotic mRNAs contain a post-transcriptionally added polyA tail that is important for many aspects of mRNA function. The polyA tail is added at the polyA site (PAS) in the nucleus in a two-step reaction consisting of a large cleavage complex that cleaves the pre-mRNA into two fragments followed by polyA tail addition to the upstream fragment (Zhao et al. 1999). Typically, PAS regulation involves the choice between two or more PAS on a single pre-mRNA (Zhao et al. 1999; Edmonds 2002) that can alter the coding region, miRNA binding sites, as well as stability and localization elements. Once considered rare, alternative PAS choice is actually prevalent with current bioinformatically based analyses of an ever increasing transcriptome database concluding that $>50 \%$ of human genes contain multiple PAS (Beaudoing and Gautheret 2001; Tian et al. 2005; Yan and Marr 2005). Genes with a single PAS can

\footnotetext{
${ }^{1}$ These authors contributed equally to this work.

Reprint requests to: Samuel I. Gunderson, Department of Molecular Biology and Biochemistry, Rutgers University, Piscataway, NJ 08854, USA; e-mail: gunderson@biology.rutgers.edu; fax: (732) 445-4213.

Article published online ahead of print. Article and publication date are at http://www.rnajournal.org/cgi/doi/10.1261/rna.756707.
}

also be upregulated or downregulated by modulating the activity of the single PAS as an "on-off" mechanism. An example of this is the U1 snRNP-specific U1A protein that negatively autoregulates itself by binding as two molecules to a polyadenylation inhibitory element (PIE) found in its own 3'UTR (Boelens et al. 1993; Gunderson et al. 1994, 1997). The regulatory mechanism involves the (U1A) $)_{2}-\mathrm{PIE}$ RNA complex inhibiting the polyadenylation step by binding to and inhibiting the activity of polyA polymerase (PAP), the enzyme that adds the polyA tail to cleaved premRNA. Without a polyA tail the U1A pre-mRNA fails to mature and is degraded by the nuclear exosome, resulting in lower levels of U1A mRNA and protein.

More recently, U1A was shown to regulate IgM heavy chain gene expression during $B$ cell differentiation by inhibition of the secretory PAS (Phillips et al. 2001). Unlike the "on-off" autoregulation, the IgM system involves the choice between two PAS, and U1A inhibits both the cleavage and the polyadenylation steps via binding to two downstream and three upstream nonconsensus sites, respectively, of the secretory PAS (Phillips et al. 2001, 2004). A differentiation-specific increase in activity of the secretory PAS is achieved by decreasing the levels of both total U1A and snRNP-free U1A, the latter a consequence of 
shifting more U1A into the U1 snRNP-bound population where it is unable to regulate polyadenylation (Phillips et al. 2001; Milcarek et al. 2003; Ma et al. 2006).

Regulation of cleavage and polyadenylation in mammals by components of the core splicing and polyadenylation machineries is a recurrent theme that is not limited to U1A. The splicing factors SRP20 and the polypyrimidine tract binding protein activate an intron 4-located PAS of the Calcitonin/CGRP gene resulting in activation of expression of the CGRP-specific mRNA (Lou et al. 1998). hnRNPF and $\mathrm{H}$ also affect the IgM secretory PAS (Arhin et al. 2002) as does artificially raising CstF64 levels (Takagaki et al. 1996; Shell et al. 2005). In vitro studies have shown the mammalian cleavage factor 1 (CFIm), via binding multiple sites upstream of the AAUAAA signal, can enhance PAS activity of a number of genes including a CFIm subunit (Venkataraman et al. 2005). As discussed next, U1 snRNP is also a potent regulator of PAS.

In mammals, U1 snRNP contains 10 proteins bound to a U1 snRNA that functions early in splicing via a basepairing interaction between U1 snRNA and the $5^{\prime}$ splice site (ss) sequence (Will and Lührmann 1997). Separate from its role in splicing, U1 snRNP/U1 snRNA binding sites (herein called U1 sites) can inhibit gene expression by inhibiting PAS activity. This was first shown in papillomaviruses where U1 sites potently inhibit expression of the viral late genes by inhibiting the late gene PAS (Furth et al. 1994), and later on it was demonstrated that U1 snRNP bound to the 5 'ss in HIV-1 inhibits the polyA signal in the HIV-1 5'LTR (Ashe et al. 2000). From these studies a potent gene-silencing technology was developed where the artificial targeting of U1 snRNP to base pair (bp) to a U1 site in the $3^{\prime}$ terminal exon of either specific reporter or cellular genes resulted in strong silencing (typically 15-30fold) of expression of that gene (Beckley et al. 2001; Fortes et al. 2003; Sajic et al. 2007). The inhibitory mechanism involves the U1-70K subunit of U1 site-bound U1 snRNP inhibiting the polyadenylation activity of polyA polymerase (Gunderson et al. 1998; Fortes et al. 2003; Sajic et al. 2007). Even if such inhibitory U1 sites match the consensus 5'ss, we choose here to call them U1 sites because they are fundamentally different in that: (1) they are not involved in splicing because there is a lack of an associated downstream 3 'ss to splice to, and (2) the U1 snRNP:U1 site duplex must be $\geq 8 \mathrm{bp}$ with an abrupt loss of activity for 7-bp duplexes (Fortes et al. 2003). In contrast, splicing-active mammalian 5 'ss sequences are highly degenerate, many of which make duplexes of $<6$ bp with U1 snRNP.

Although U1 sites cause a reduction in mRNA levels of their target gene, they are highly distinct from traditional mRNA stability elements in three ways. First, U1 sites block biosynthetic maturation of a gene-specific pre-mRNA in the nucleus, whereas mRNA stability elements influence stability of a preexisting cytoplasmic pool of a gene-specific mature mRNA. Second, U1 sites only inhibit when placed in the 3' terminal exon (Beckley et al. 2001; Fortes et al. 2003), a restriction not applicable to mRNA stability elements (Guhaniyogi and Brewer 2001; Wilusz et al. 2001). Third, U1 sites do not inhibit expression of mRNAs having a $3^{\prime}$ end histone stem-loop element in place of the polyA signal (Fortes et al. 2003), whereas mRNA stability elements destabilize both types of mRNA (Pettitt et al. 2002).

To date, naturally existing U1 sites have only been reported in papillomaviruses (Furth et al. 1994; Cumming et al. 2003) with no functional ones being reported in cellular genes. Here, we use bioinformatics and a reporter gene and in vitro polyadenylation assays to identify and characterize a U1 site in the terminal exon of the human U1A gene. The U1 site is within a conserved bipartite element that represses $\mathrm{U} 1$ site activity by base pairing to and trapping U1 snRNP in an inactive conformation. In its natural context the U1 site/bipartite element synergizes with the nearby PIE to negatively regulate U1A expression. This is the first functional U1 site to be identified in a cellular gene and of a synergistic action between two PAS regulatory elements.

\section{RESULTS}

\section{Identification of a conserved $\mathrm{U} 1$ site in mammalian U1A gene 3 'UTRs}

To identify genes with $\mathrm{U} 1$ sites we searched a number of 3'UTR databases, ultimately focusing on one located at http://bighost.area.ba.cnr.it/BIG/UTRHome (Pesole et al. 2002) because: (1) it is well curated in that redundant sequences and cloning artifacts have been, for the most part, removed, and (2) it is searchable with very short, query sequences $(<15$ nucleotides $[\mathrm{nt}])$ with PatSearch software that, unlike standard BLAST search programs, will permit short (8-10-nt) query sequences with mismatches. Positive hits were those $3^{\prime}$ UTRs with a $\geq 8 / 10$ uninterrupted match to the consensus $\mathrm{U} 1$ site sequence CAGGUAAGUA and where the match is conserved in the 3'UTRs from $>2$ species. To our surprise the U1A gene, whose autoregulatory system we have extensively characterized in the past, was one of our top hits. The entire list of hits will be presented elsewhere (S.I. Gunderson, R. Goraczniak, and E.S. Ho, unpubl.). Here we wanted to analyze in detail the U1A gene so as to demonstrate whether the U1 site is indeed active. Mammalian U1A 3' UTRs were aligned by ClustalW (Thompson et al. 1994) and divided into four regions (Fig. 1), with region A having $15 / 56$ nt $(\sim 27 \%)$ identical in all seven species. Region B has $36 / 44 \mathrm{nt}(\sim 82 \%)$ identical and contains an $8 / 10$ match to the consensus U1 site that can make eight uninterrupted base pairs with U1 snRNA, the minimal number needed for polyadenylation inhibition (Beckley et al. 2001; Fortes et al. 2003). Region C is only moderately conserved with $11 / 25 \mathrm{nt}$ (44\%) being identical. Region D, containing the highly 


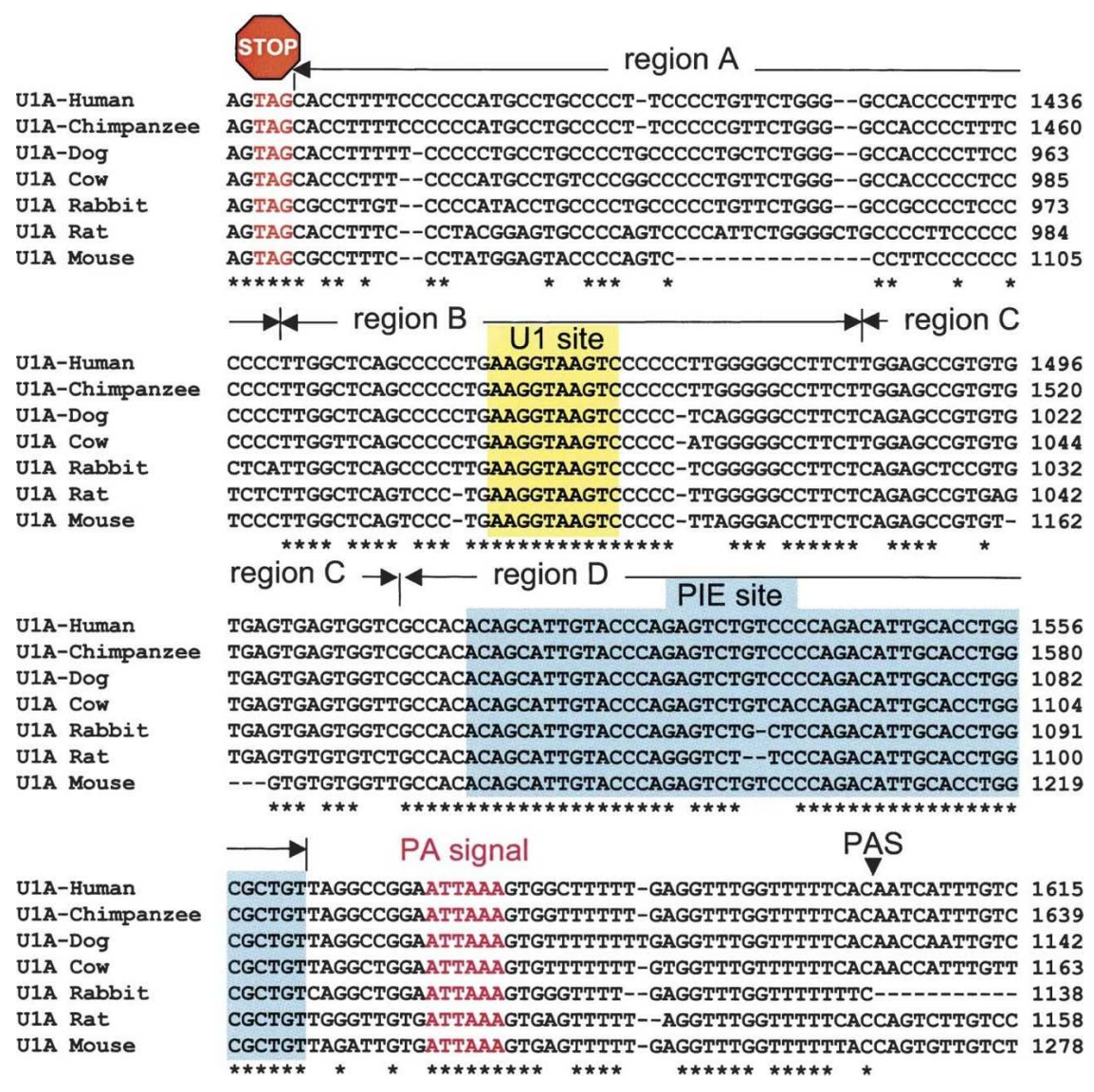

FIGURE 1. The U1 site region is conserved in mammals. Shown is a ClustalW alignment of seven mammalian U1A 3'UTRs: human (NM_004596), chimpanzee (XM_512674), dog (XM_533663), cow (BC112544), rabbit (AY387676), rat (NM_001008303), and mouse (BC003229). The numbers on the right are based on the accession numbers. The sequences span from the stop codon TAG (in red) to the polyA site (PAS) (the human U1A PAS is labeled with an arrowhead), plus several nucleotides of the genomic sequence past the cleavage site (except for rabbit U1A, whose genome sequence is not available). The boundaries of regions A-D are labeled with arrows ending in a vertical bar, the putative U1 sites are highlighted with yellow, the PIE sites with green, and the AUUAAA poly(A) signals with dark pink. ${ }^{\star}$ Means identical nucleotides. A ClustalW alignment of sequences downstream from the PAS showed no significant conservation (data not shown).

characterized PIE region, is the most conserved with $48 / 53$ nt $(\sim 91 \%)$ identical, with this conservation already being noted when U1A autoregulation was first discovered (Boelens et al. 1993). Strikingly, the distances between the $\mathrm{U} 1$ and PIE sites and the U1 and AUUAAA sites are also well conserved. This additional conservation of position made this putative U1 site unique among the top hits in our search and prompted us to determine whether it is functional. So why was the conserved pattern in regions $\mathrm{B}$ and C not reported in 1993? First, U1 sites had not yet been discovered, and second, the only known U1A genes in 1993 were in human, mouse, and Xenopus. Regions B and C are missing in Xenopus, whereas mouse and human 3'UTRs are too similar to make any sequence comparisons meaningful. Indeed, additional bioinformatic analysis of lower vertebrate U1A genes (e.g., Xenopus laevis and fish) shows that regions $\mathrm{B}$ and $\mathrm{C}$ and the $\mathrm{U} 1$ site itself are not conserved, whereas region $\mathrm{D}$ (the PIE site) is highly conserved (Supplemantal Material; data not shown) Thus, the U1 site conservation in regions $\mathrm{B}$ and $\mathrm{C}$ is restricted to mammalian U1A genes.

\section{FLAG-tagged U1A cDNA expression plasmids}

To match as closely as possible the endogenous human U1A gene, U1 site function was tested in the context of the full-length natural human U1A mRNA by transfection of a Flag-tagged U1A cDNA expression plasmid under the control of a constituitive promoter (Fig. 2A). Six matching plasmids were produced, each containing one of various combinations of wild-type (wt), mutant (mt), or up-mutant (up) U1 sites with either a wt or mt PIE. The upU1 site is a perfect $10 / 10$ match in place of the naturally occurring $8 / 10$ match to the consensus binding site. The mtU1 and mtPIE sites were previously shown to be inactive for polyadenylation inhibition (Boelens et al. 1993; Fortes et al. 2003).

\section{The U1 and PIE sites synergize but the $U 1$ site has no activity when PIE is mutated}

Transfected cells were analyzed by Western blotting (an example is shown in Fig. 2B), the signals quantitated with ImageQuant 5.2 software and the results graphed (Fig. 2C) as inhibitory activities based on the following calculation. First, the FlagU1A signal was normalized by comparison with the cotransfected FlagPRP28 signal. Second, the double-mutant $\mathrm{mtU} 1 / \mathrm{mtPIE}$ plasmid had the highest expression level as it lacks inhibitory elements and so its inhibitory activity was set to 1.0 as the reference plasmid. Finally, dividing the normalized signal of the $\mathrm{mtU} 1 / \mathrm{mtPIE}$ reference plasmid by the test plasmid gave the fold inhibition. For example, the $\mathrm{mtU} 1 / \mathrm{wtPIE}$ plasmid was 3.0-fold less expressed than the $\mathrm{mtU} 1 / \mathrm{mtPIE}$ plasmid; thus, the mtU1/wtPIE plasmid and consequently its PIE site has a 3.0-fold inhibitory activity, a value consistent with the previously reported 3.1-fold inhibitory activity in vivo when PIE was inserted into a reporter plasmid (Boelens et al. 1993). To confirm that the Western blot signal is dose dependent, a titration of HeLa nuclear extract (NXT) 


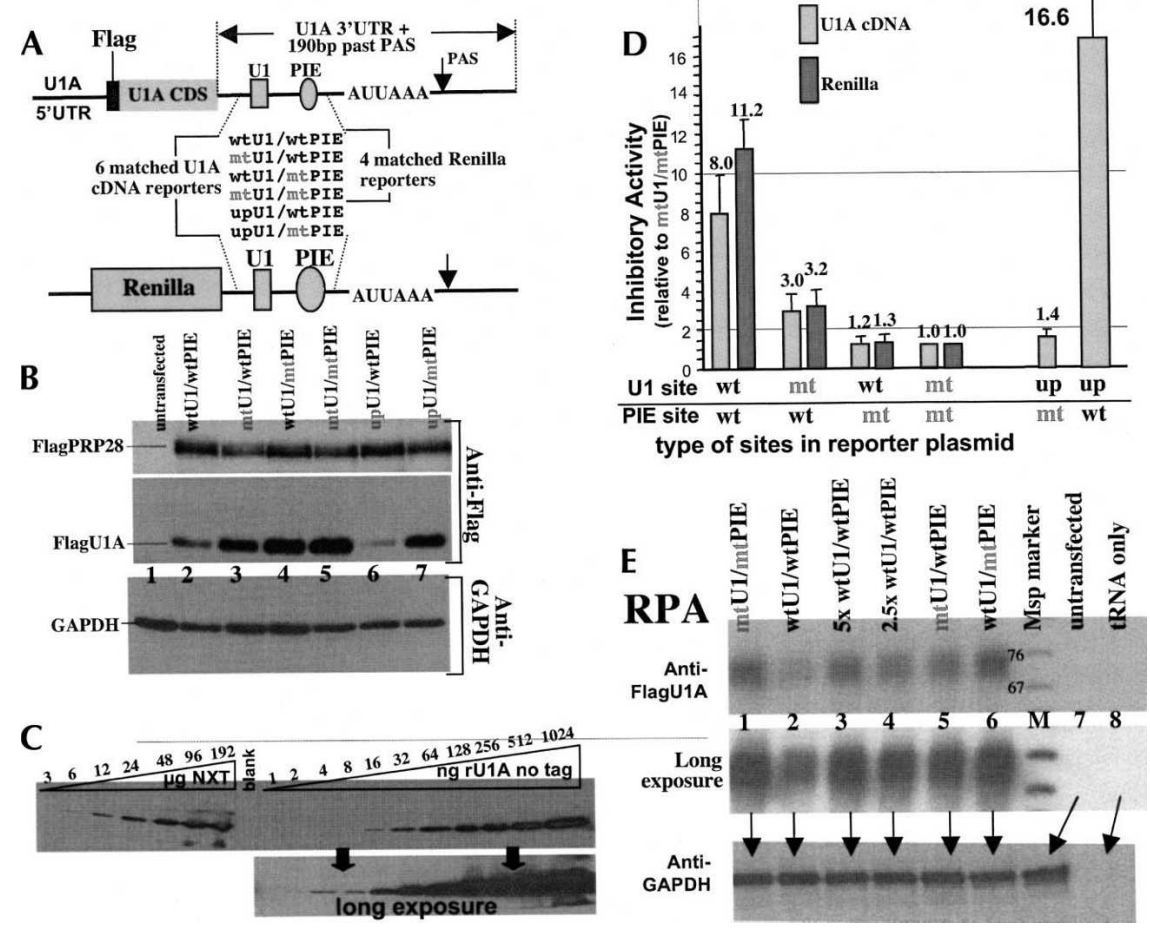

FIGURE 2. Inhibitory activity of the U1 and PIE sites. (A) The six U1A cDNA plasmids contain a chicken actin promoter driving expression of the full-length human U1A cDNA where the 3' UTR has one of various combinations of the wild-type (wt), mutant (mt), or up mutant (up) U1 site combined with a wt or mt PIE site. The FLAG tag serves to distinguish transfected from endogenous $\mathrm{U} 1 \mathrm{~A}$ and was placed at the $\mathrm{N}$ terminus, as this inactivates the $\mathrm{N}$-terminal RNA binding domain, thus producing a U1A protein unable to autoregulate either the transfected U1A expression plasmid or the endogenous U1A gene (Gunderson et al. 1997). To more closely match the endogenous gene, $190 \mathrm{bp}$ of human genomic U1A gene sequence starting from the natural PAS to $190 \mathrm{bp}$ downstream were inserted into the corresponding PAS position of the cDNA plasmid. Four Renilla reporter plasmids (Promega) with a CMV promoter were modified by replacing the $3^{\prime} \mathrm{UTR}$ and polyadenylation signal sequences with the corresponding sequences from the U1A cDNA including $190 \mathrm{bp}$ past the PAS. (B) Western blot. Each U1A cDNA plasmid $(0.5 \mu \mathrm{g})$ in panel $A$ was transfected into four Million Hela-Tet cells along with $0.5 \mu \mathrm{g}$ Flag-PRP28 expression construct that controls transfection efficiency. Hela-Tet cells from the "Tet-Off" system were used because the FlagPRP28 reporter is under the control of a Tet-responsive promoter. After $48 \mathrm{~h}$, cells were lysed in Laemmli buffer and 10 $\mu \mathrm{g}$ of protein loaded on a protein gel for Western blotting followed by sequential probing with anti-Flag (upper two panels are taken from the same exposure) and anti-GAPDH (lower panel) antibodies as shown. The blot was then probed with anti-U1A antibody (data not shown). (C) The dynamic range of the Western blotting protocol was analyzed by performing a serial dilution of HeLa NXT and of purified recombinant $(r)$ untagged U1A. The blot was probed with anti-U1A856 antibody. (D) Western blot results from eight independent transfection experiments were quantitated and summarized as a graph with the inhibitory activity of the double mutant being set to 1.0. Also shown are results of eight independent transient transfections of each Renilla reporter where Renilla activity was normalized to a cotransfected Firefly reporter with the inhibitory activity of the double mutant being set to 1.0. The relative inhibitory activities were determined as described in the text. (E) RPA analysis of total RNA from transfected cells was used to measure mRNA levels produced from the U1A cDNA plasmids. The autoradiograph is representative of the data we obtained when analyzing total RNA from other independent transfections. The ${ }^{32} \mathrm{P}$-labeled anti-Flag probe specifically recognizes the Flag tag in the U1A cDNA but not that of the FlagPRP28 plasmid. The protected RPA product for the anti-Flag probe runs as a broad series of bands from 70 to $75 \mathrm{nt}$ because the 3' end is AU rich and so is "nibbled" back by the Rnase digestion step. Also shown is a longer exposure of the anti-Flag probed RPA gel. Lanes 1,2, 5, 6, and 7 are as in B. Lanes 3 and 4 are total RNA from cells transfected with $5 \times$ and $2.5 \times$ as much wtU1/wtPIE plasmid so as to assess the response of the assay to increasing amounts of mRNA. The same total RNA samples were also probed on a different gel with ${ }^{32} \mathrm{P}$-labeled anti-GAPDH as a control. Lane $M$ is a size marker of ${ }^{32} \mathrm{P}$-labeled Msp1-digested pBR322 with the 67-nt and 76-nt length bands indicated. compared to recombinant (r) U1A was done (Fig. 2D). All of the Western blot analyses used throughout this work were done in this range.

Mutation of the U1A U1 site in its natural context reduced inhibitory activity from eightfold to threefold (a 2.7-fold decrease). We were therefore surprised that the U1 site showed no (1.2-fold) statistically significant inhibitory activity when PIE was mutated. This lack of inhibitory activity sharply contrasted with previous work by us (Fortes et al. 2003) and others (Furth et al. 1994; Beckley et al. 2001) that single U1 sites inserted into reporter gene 3'UTRs strongly inhibited expression ranging from $15-$ to 32 -fold. In those publications a wide variety of reporter genes were used, including BetaGalactosidase, Chloramphenicol Acetyl Transferase, Firefly, and Renilla luciferase, GFP, YFP, RFP, as well as different reporter $3^{\prime}$ UTR and polyA signal sequences (e.g., Adenovirus, SV40, bovine growth hormone gene), indicating U1 sites maintain inhibitory activity in a variety of sequence contexts. One explanation, that such low activity is due to suboptimal base-pairing of U1 snRNA with the U1A U1 site, was ruled out for two reasons. First, the exact same 8/10 U1 site gave an 18-fold inhibition in a luciferase reporter context (Furth et al. 1994), and second, replacing the U1 site in wtU1/mtPIE with a perfect 10/10 up-mutated U1 site (upU1/mtPIE) still gave only 1.4-fold inhibition. Notably, the upU1 site, like the wtU1, could also synergize with the wtPIE site. Results statistically the same as in Figure 2C were observed when the FlagPRP28 control plasmid was omitted or when the Flag tag on U1A was replaced with an HA tag indicating neither had an effect (data not shown). Furthermore, transfection of HeLa cells in place of HeLa-Tet and transfection of various amounts of each construct gave similar inhibitory activities, the latter demonstrating endogenous factors in vivo, such as U1A and U1 snRNP, were not limiting (data not shown). Thus, the U1 site only is active in conjunction with a wtPIE site, as alone it has little or no detectable activity. 


\section{The U1A coding and 5'UTR sequences are dispensible}

To determine whether the U1A coding and 5'UTR sequences affect expression we replaced them with the Renilla coding region and reporter 5'UTR. Compared to the multistep Western blot method, the Renilla system is simple to use, has a much more rigourous and accurate normalization method, and has a far better dynamic range to measure protein expression levels, and hence, inhibitory activities. The activity of each Renilla plasmid was normalized to a cotransfected control Firefly plasmid and the inhibitory activity of the double mutant RL-mtU1/mtPIE was set to 1.0. As shown in Figure 2D, the pattern of inhibitory activity was nearly identical to that of the full-length cDNA plasmids, indicating the coding and 5'UTR regions of the U1A mRNA do not affect the inhibitory activity of the $3^{\prime} \mathrm{UTR}$.

\section{The U1 and PIE sites lead to reduced mRNA levels}

As previously shown, U1 and PIE sites in other reporter gene contexts inhibit by reducing mRNA levels (Boelens et al. 1993; Fortes et al. 2003). To confirm this is the case here, total RNA from HeLa cells transfected with the various cDNA plasmids were analyzed by ribonuclease protection assay (RPA) where the RPA probe anneals to the 60-nt Flag epitope tag in U1A but not to the Flag tag in the FlagPRP28 control or to endogenous U1A (Fig. 2D). Parallel RPAs with an anti-GAPDH probe were done to control that the quality and amount of RNA used were the same. As can be seen in Figure 2E, the effect on mRNA levels by the U1 and PIE sites closely tracks the protein levels seen in Figure 2D. Although reduced mRNA levels are likely a consequence of inhibiting mRNA biosynthesis by inhibiting polyA site activity, as this is consistent with prior work on each element, it was proposed that the juxtaposition of the U1 and PIE sites together in the natural U1A cDNA would affect mRNA stability. However, we ruled this out because actinomycin treatment of cells transiently transfected with the various U1A cDNA plasmids, followed by quantitative PCR analysis, indicated mRNA stability does not change (data not shown).

\section{The isolated U1A U1 site strongly inhibits Renilla expression}

As mentioned above, we unexpectedly found that the U1A site has no activity on its own and only modest activity when PIE is wild type. In order to understand these unexpected results, we reduced the $3^{\prime} \mathrm{UTR}$ of RL/wtU1/wtPIE to a minimum to make the RLm plasmid ( $m=$ minimal) where sequences necessary for polyA site activity were retained while the upstream regions A, B, C, and D were deleted (Fig. 3A). As RLm lacks inhibitory elements, we set its inhibitory activity to 1.0 as the reference plasmid. Regions A, B, and C had no intrinsic inhibitory activity when each was inserted into RLm (data not shown). Insertion of wtPIE (region D) to make RLm/ wtPIE decreased expression 2.8-fold relative to insertion of a mutated PIE (RLm/mtPIE) indicating PIE has a 2.8 -fold inhibitory activity, nearly the same value as it had in Figure 2. In contrast, insertion of just part of region $B$, namely the U1A U1 site (10 nt), to make RLm/wtU1/wtPIE and RLm/ wtU1/mtPIE, caused a 12 -fold repression both when PIE was wild type (12.2 divided by 1.00) and when PIE was mutated (33.4 divided by 2.8). This 12 -fold effect was specific as two matching control plasmids with a mutated U1 site gave no effect. The 12 -fold magnitude of inhibition is consistent with what we and others have observed for inhibition mediated by the $5^{\prime}$ end U1 snRNA base-pairing to a $\mathrm{U} 1$ site. To rigorously demonstrate that such base-pairing is
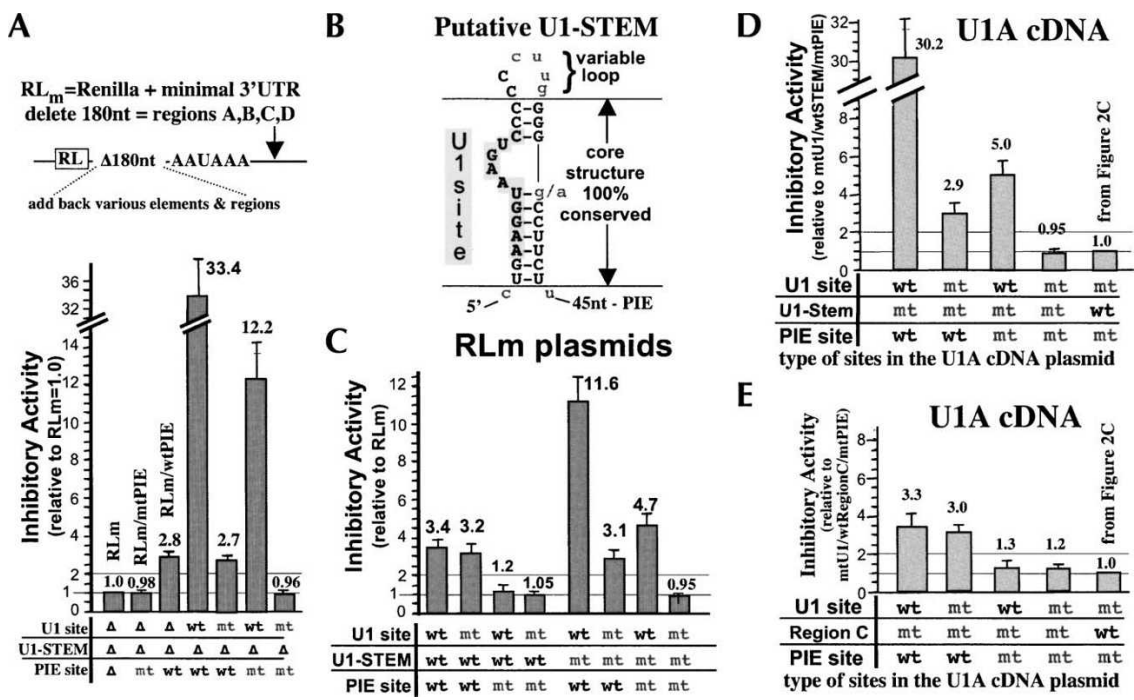

FIGURE 3. The U1 site is suppressed by base-pairing to a U1-STEM, and region C contributes to synergy. (A) RLm is a deletion mutant of the Figure 2A Renilla reporter that retains the minimal core polyA signal sequences while deleting upstream regions A, B, C, and D. Each upstream region was then reinserted into RLm either alone or in combination with other regions and the inhibitory activity measured by transfection as in Figure 2. The plasmids were analyzed as described in Figure 2. As shown, the U1A U1 site has high inhibitory activity, suggesting that flanking sequences present in Figure 2, but absent here, are repressing U1 site activity. (B) Conservation in mammalian U1A genes of base-pairing between the U1-STEM and the U1 site. The specific sequence shown is from human U1A. The C:G of the variable loop is shown to not be base paired because it is not conserved in two of the seven mammalian U1A genes as shown in Figure 1. Perfectly conserved nucleotides are in uppercase and nonconserved nucleotides in lowercase. $(C)$ Analysis of the U1-STEM or a mutated U1-STEM when inserted into the four U1-site-containing plasmids in Figure 3A. (D) Analysis of the U1-STEM mutation $(D)$ and the region $C$ mutation $(E)$ in the context of the U1A cDNA plasmid. The assays and analysis were done as in Figure 2. 
occurring in vivo, we expressed a compensatory or "suppressor" U1 snRNA that has its 5 ' end complementary to the mutated U1 site. If the suppressor U1 snRNA restores inhibition to a reporter with such a mutated U1 site then we can conclude inhibition is mediated by base-pairing to U1 snRNA. Such suppressor U1 snRNA experiments have been done by us and others for reporter genes as well as for the papillomavirus late genes (Furth et al. 1994; Beckley et al. 2001; Fortes et al. 2003). Expression of a suppressor U1 snRNA restored inhibitory activity to the mutant U1 site, demonstrating base-pairing of the $5^{\prime}$ end of U1 snRNA to the U1 site is required for inhibition (data not shown).

\section{A bipartite element consisting of a U1 site repressed by a U1-STEM structure}

Next, we decided to determine why the U1A U1 site in Figure 3A was now highly inhibitory and its activity independent of PIE. By use of reporter assays, we recently demonstrated that a highly active U1 site could be fully repressed when completely base-paired within a stem structure (Fortes et al. 2003). Inspection of the natural U1A 3'UTR identified a putative secondary structure in region $\mathrm{B}$, here designated the $\mathrm{U} 1-\mathrm{STEM}$ (Fig. 3B), where the upstream half of the U1 site is base-paired to the U1STEM while the downstream half is in an exposed loop. Strikingly, the putative base-pairing pattern is perfectly conserved in all seven mammals (Supplemental Material; data not shown), and these sequences are the most conserved part of the sequences flanking the U1A U1 site. To test the role of the U1-STEM, 18 nt of the wild-type U1-STEM (wtSTEM) were inserted into the four U1-sitecontaining plasmids shown in Figure $3 \mathrm{~A}$ and the four resulting plasmids tested for activity in transfected cells. Pairwise comparison of Panel A with Panel C plasmids indicated the 18-nt wtSTEM caused a 10-fold repression when PIE was wild type (33.4-fold to 3.4-fold) and when PIE was mutant (12.2-fold to 1.2-fold). This repression was specific to the U1 site because the wtSTEM had no effect on PIE activity (still $\sim 3$-fold) and had no activity on its own (compare RLm/mtU1/mtPIE to RLm/mtU1/wtSTEM/ mtPIE). To demonstrate base-pairing was the basis of repression, as opposed to changing the spacing between elements, four additional plasmids were made where an 18-nt mutated U1-STEM (mtSTEM) sequence was inserted where the mutation should significantly reduce base-pairing potential. The mtSTEM had no activity on its own, and did not affect PIE activity which was still $\sim 3$-fold. As predicted, the mtSTEM restored U1 site activity both when PIE was wild type (11.6-3.4-fold) and when PIE was mutated (4.7-1.2-fold), thus strongly supporting the U1STEM:U1 site base-pairing model. Although this restoration was specific, its magnitude was not quite at the level of the Figure 3B plasmids, which have a deleted U1-STEM.
This suggests that the mtSTEM could still have residual base-pairing to the U1 site. Interestingly, the pattern of the wtSTEM-containing plasmids in Figure 3C compared to the Renilla plasmids in Figure 2C indicate synergy between the U1 and PIE sites is lost. Insertion of region C (but not region A) restored this synergy to the levels seen in Figure 2C (Supplemental Material; data not shown). Thus, we have identified (1) a bipartite U1 element comprised of a highly inhibitory $\mathrm{U} 1$ site being repressed via base-pairing to a U1-STEM, and (2) that region C is needed for synergy between the bipartite U1 element and PIE.

\section{The bipartite element and region $C$ have the same activity in the context of the U1A mRNA}

To test whether the U1-STEM represses in the context of the natural U1A mRNA, four plasmids were made that identically match the four Figure 2A U1A cDNA plasmids (we did not include the two upU1 site plasmids) except the U1-STEM was mutated with the same mutation as in Figure 3C. An additional set of four U1A cDNA plasmids were made where region $C$ was mutated rather than the U1-STEM. All eight plasmids were analyzed by transfection as in Figure 2, and the results are graphed in Figures 3D (U1-STEM mutants) and 3E (region C mutants). Pairwise comparison of the plasmids in Figure 2C with those in Figure 3D-E indicate that by themselves the U1-STEM and region $\mathrm{C}$ have no activity and do not affect the activity of PIE, which remained at threefold. In contrast, mutation of the U1-STEM specifically de-repressed the activity of the U1 site both when PIE was mutated (1.2-fold in Fig. 2C to 5.0-fold) or wild type (eightfold in Fig. 2C to 30.2-fold). Mutation of region $\mathrm{C}$ specifically caused a loss of synergy between the U1 and PIE sites. We conclude that both the U1-STEM and region C function in the full-length U1A mRNA, have no inhibitory activity on their own, and have no effect on PIE. Furthermore, the U1-STEM specifically represses the $\mathrm{U} 1$ site while region $\mathrm{C}$ specifically affects synergy.

\section{Synergy can be reconstituted in HeLa NE}

To elucidate the mechanism of inhibition seen in vivo we performed in vitro cleavage and polyadenylation assays in HeLa nuclear extract (NXT) with RNA substrates derived from the U1A 3'UTR and polyA site region extending from region $\mathrm{B}$ to $80 \mathrm{nt}$ past the PAS (see Fig. 5A for a schematic of the RNA). This assay system was chosen as it contains all the factors necessary to reconstitute U1A:PIE autoregulation by itself and U1 snRNP:U1 site-mediated polyadenylation inhibition by itself. Our previous work showed U1Amediated inhibition requires addition of recombinant (r) U1A (Boelens et al. 1993) because nearly all ( $\sim 95 \%)$ of endogenous U1A is in the U1 snRNP, and therefore, is unavailable to bind PIE (Boelens et al. 1993; Ma et al. 
2006). The various rU1As used in Figures 4 and 5 were judged to be pure based on Coomassie-stained gels and Western blotting (Supplemental Material; data not shown). Inhibition of cleavage/polyadenylation of the wtU1/wtPIE RNA was seen upon addition of $5 \mathrm{ng}$ of rU1A (Fig. 4A, lane 3 ) and increased amounts of rU1A gave increased inhibition. If the inhibitory activity of the (U1A) $)_{2}$ PIE complex on the wtU1/wtPIE RNA is independent of the U1 site then we would expect the mtU1/wtPIE RNA to have the same inhibitory response to added rU1A. Instead, $12.5 \mathrm{ng}$ of U1A were needed to observe inhibition of the mtU1/wtPIE RNA (Fig. 4A, lane 11), indicating that the wtU1 site strengthens the rU1A-mediated inhibition. Most importantly, this increase in inhibition correlates well with the 2.7-fold difference in synergistic inhibitory activity that the U1 and PIE sites exhibit in vivo (Fig. 2) indicating synergy has been reconstituted with this in vitro assay. Additional controls underscored the specificity of these results as addition of rU1A did not inhibit the wtU1/mtPIE RNA (Fig. 4A, lanes 16-18) or the $\mathrm{mtU} 1 / \mathrm{mtPIE}$ RNA (data not shown).

To understand the basis of the synergy we added up to a 50 -fold stoichiometric excess of purified U1 snRNP over RNA substrate to these assays and saw no effect, indicating U1 snRNP is not limiting (data not shown). To eliminate base-pairing by U1 snRNP we employed a well-known method where NE is pretreated with a U1 oligo complementary to nt $1-12$ of U1 snRNA (Krämer and Keller 1990). Rnase H intrinsic to NE degrades the RNA strand of the RNA:DNA duplex, that is, nucleotides $1-12$ of U1 snRNA are removed, thereby preventing U1 snRNP:U1-site base-pairing. As a control, NE was also pretreated with an unrelated oligo (nonspecific oligo). As shown in the Northern blot in Figure $4 \mathrm{~B}$, the U1-oligo quantitatively and specifically removed $\sim 10$ nt off of $U 1$ snRNA. In Figure 4B, lanes 4-9, we reproduce published data where U1oligo treatment strongly de-represses polyadenylation of an Adenovirus L3 polyadenylation substrate RNA that artificially contains a U1 site (AdL3+ wtU1) (Gunderson et al. 1998). Two controls demonstrated that the derepression is specific. First, the U1 oligo had no effect on a matching substrate having a mutated $\mathrm{U} 1$ site (AdL3+ mtU1). Second, mock treatment with a nonspecific oligo had no effect. We then used these oligo-treated NEs to analyze the U1A RNA substrates. In contrast to the AdL3+wtU1 RNA, polyadenylation of the wtU1/wtPIE U1A RNA was not de-repressed, as illustrated by the inhibition profile of U1 oligoand nonspecific oligo-treated reactions, which were the same (Fig. $4 \mathrm{C}$, cf. lanes $2,9,16$, and 22). However, the U1 oligo treatment did cause loss of synergy (Fig. $4 \mathrm{C}$, cf. lanes 16-21 and 22-27) compared to the nonspecific-oligo-treated reactions (Fig. 4C, cf. lanes $2-7$ and 912). Thus, we can reconstitute synergy in vitro by addition of rU1A to HeLa $\mathrm{NE}$, and we find that synergy requires the 5 ' end of U1 snRNP and the U1 and 
A

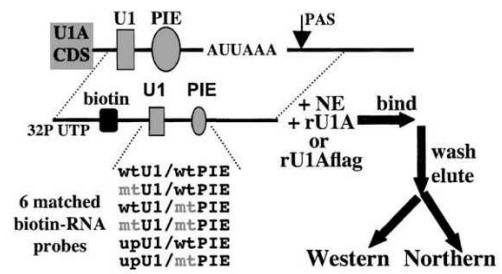

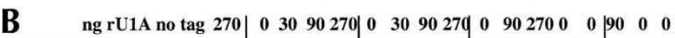
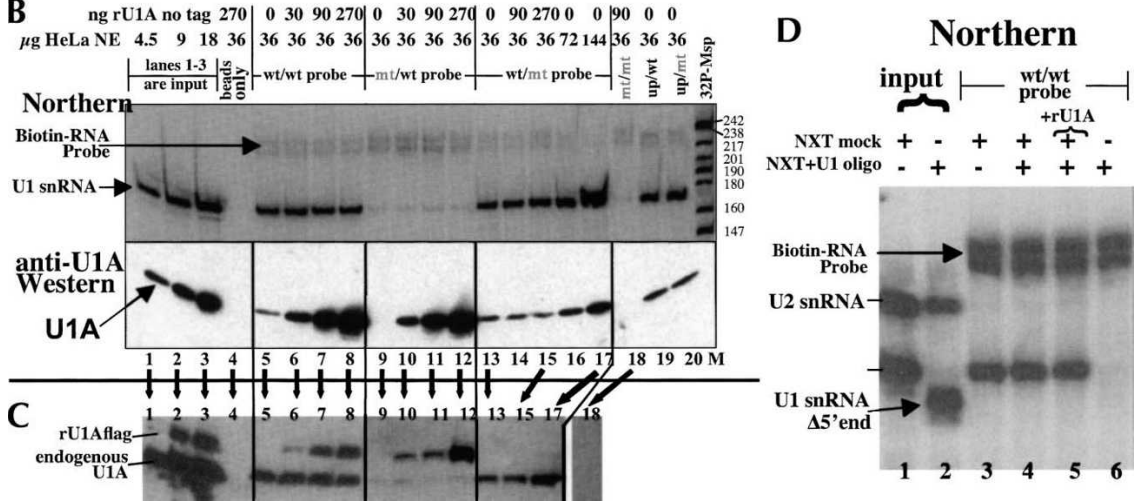

FIGURE 5. U1A and U1 snRNP do not significantly affect each other's binding in HeLa NE. (A) Schematic of a biotin-selection protocol that is described in the text. The RNAs are double labeled with ${ }^{32} \mathrm{P}-\mathrm{UTP}$ and biotin (black square) and are derived from the U1A $3^{\prime} \mathrm{UTR}$. $(B)$ Shown are the Northern and Western blot results of the biotin selection assay. The type of double-labeled biotin-RNA probe and the amount of rU1A and NE added to each reaction are indicated. As described in the text, these double-labeled probes also appear on the Northern blot and serve as an internal control. To detect the amount of unlabeled U1 snRNP in the pulldown, the Northern blot was probed with an anti-U1 snRNA probe. The Western blot was probed with an anti-U1A antibody. Lanes 1-3 are NE inputs showing the linear response of both the Northern and Western blots in this range. Lane 4 shows the background binding to beads without any RNA probe. The amounts of NE and exogenously added rU1A are indicated. Also indicated are the positions of the double-labeled RNA probes, U1 snRNA and U1A. The size marker lane is as in Figure 4. The entire experiment was repeated $5 \times$ with results consistent to the example shown here. $(C)$ Panel $B$ was repeated but rU1Aflag was used in place of rU1A. Three times less rU1Aflag was used so that its Western blot signal would not mask that of endogenous U1A and film exposures were longer to help visualize the weak endogenous U1A signal, especially in lanes 9-12. Lane 18 was spliced in from a different part of the same autoradiograph. $(D)$ The biotin assay was done with HeLa NE either mock treated with a nonspecific oligo (lane 1) or U1 oligo treated to remove the $5^{\prime}$ end of U1 snRNA. Lane 1 contains twice as much RNA as lane 2 so as to confirm the assay is dose dependent. The Northern blot was probed with anti-U1 and anti-U2 snRNA probes. Lanes 4 and 5 are controls where the mock-treated and U1 oligo-treated reactions were mixed just prior to selection in order to show the U1 oligo does not affect the biotin selection step. Lane 5 also included $270 \mathrm{ng}$ rU1A during the incubation step with the biotinylated RNA. The Northern blot shows U1 oligo treatment caused complete loss of binding of U1 snRNA to the wt/wt biotin probe compared to mock treatment. Results were the same for the other three types of probes (data not shown).

PIE sites. Next, we examined the binding of these factors to the RNA.

\section{Synergy is not based on enhanced binding of U1 SnRNP or U1A to the $3^{\prime}$ UTR}

One straightforward model to explain synergy is that the U1-STEM occludes binding of U1 snRNP to the U1 site, and that the U1A:PIE complex overcomes this occlusion. To test this, biotin-selection ("pull-down") assays were done using the same RNA probes as in Figure 4 except that they were double labeled with biotinCTP and ${ }^{32}$ P-UTP (Fig. 5). BiotinU1A-RNA probes bound to streptavidin beads were mixed with HeLa NE and zero or increasing amounts of recombinant U1A (rU1A) protein. After wash steps, specifically bound protein(s) and/or RNAs were eluted with SDS buffer and analyzed by Western and Northern blotting (Fig. 5B). The U1A Western blot signal corresponds to exogenously added rU1A+U1 snRNPbound U1A+snRNP-free-U1A in the NE. The Northern membrane was probed with ${ }^{32} \mathrm{P}$-anti-U1 snRNA to measure the amount of U1 snRNP pulled down. The Northern blot also contains signals from the ${ }^{32} \mathrm{P}$-labeled, biotin-RNA probe that coeluted from the beads which controlled for: (1) uniform transfer of RNAs to the Northern membrane, and (2) that similar amounts of the U1A RNA probe were used for each pull down and that the RNA had not been degraded during all of the incubation, wash, and elution steps. Increasing both NE (Fig. 5B, lanes 15-17) and rU1A (Fig. 5B, lanes 5-12) gave increased signals, demonstrating that the U1A RNA probe was in stoichiometric excess over both U1A and U1 snRNP, a requirement for detecting changes in binding. Specificity of binding was confirmed as mutation of the U1 site (Fig. 5B, lanes 9-12) and both sites (Fig. 5B, lane 18) resulted in a significant reduction in binding. Probing the Northern to detect other spliceosomal U snRNAs (U2, U4, U5, and U6) demonstrated their binding was very low to not detectable (for an example, see U2 snRNA in Fig. 5D). The $\mathrm{mtU} 1 / \mathrm{wtPIE}$ probe (Fig. 5B, lane 9), but with no added rU1A, gave a visible Western blot signal only upon longer exposures consistent with previous data that the amount of snRNPfree U1A in HeLa NE is relatively low (Boelens et al. 1993).

Having established that the assay conditions were both specific and in probe excess, the key question could be addressed, namely does increasing the U1A:PIE complex lead to changes in the levels of U1 site-bound U1 snRNP. As expected, increasing amounts of rU1A added to the wtU1/wtPIE probe gave an increasing Western blot signal of U1A; however, the Northern blot shows no change of U1 snRNA bound to the probe (Fig. 5B, lanes 5-8). 
This indicates that (1) the amount of probe is in excess over the U1A protein, and (2) the binding of rU1A to the PIE site has no effect on the binding of U1 snRNP to the U1 site. Likewise, if U1 snRNP is affecting binding of U1A to PIE, then the Western blot signals in lanes 6-8 (Fig. 5B) would be either increased or decreased relative to lanes 10-12 (Fig. $5 B)$. Instead, quantitation of these signals, which included normalization with the eluted RNA probes shown on the Northern blot panel, indicates there is no difference. Finally, the signals in lanes 5 and 13 (Fig. 5B) are of the same intensity, indicating that the presence or absence of the PIE site has no detectable affect on U1 snRNP binding. Thus, these data support the conclusion that synergy is not due to enhanced binding of U1 snRNP or U1A to the $3^{\prime}$ UTR.

To clearly distinguish rU1A from endogenous we repeated these experiments with a C-terminal Flag-tagged rU1A protein (rU1Aflag) as shown in Figure 5C. The various rU1A preparations were judged to be nearly $100 \%$ pure, and Western blots titrating recombinant U1A versus endogenous U1A in NE were done to calibrate the amounts of protein and to avoid interfering of their Western blot signals (Fig. 2B, lower panel). As can be seen, most of the endogenous U1A signal is coming from U1 snRNP-bound U1A (Fig. 5C, cf. lanes 9-12 and 13-15). Northern blotting showed addition of rU1Aflag had no effect on the U1 snRNA signal (data not shown), which is in agreement with the Northern blot in Figure 5B. To demonstrate that the specificity of U1 snRNP binding we removed the $5^{\prime}$ end of U1 snRNA and observed that U1 snRNP binding is completely absent (Fig. 5D). In closing, we note the striking lack of correlation between binding of U1 snRNP to the U1 site and the inhibitory activity measured in vivo. In particular, the upU1/wtPIE plasmid gave a 16 -fold inhibitory activity in vivo compared to the $\mathrm{wtU} 1 / \mathrm{mtPIE}$ (1.2-fold) or the wtU1/wtPIE (eightfold) plasmids (Fig. 2). Nevertheless, all three RNA substrates bound U1 snRNP with approximately the same efficiency (Fig. 5B). Furthermore, we had expected the U1-STEM to inhibit U1 snRNP binding; however, biotin pull-down assays comparing RNA substrates with a wtSTEM to those with a mtSTEM showed no difference in U1 snRNP binding (data not shown). As described below, we propose a model where U1 snRNP is bound to the wild-type bipartite element but is unable to inhibit unless the PIE site is wild type and occupied by U1A.

\section{DISCUSSION}

An evolutionarily conserved 10-nt binding site for the U1 snRNP (U1 site) was identified in the U1A gene's 3'UTR and shown to function in repressing U1A expression. The $\mathrm{U} 1$ site is within a bipartite element that also contains a U1-STEM that base pairs to and represses the activity of the U1 site. This is the first example of an endogenous cellular gene having a functional U1 site. Up to now, only two types of U1 sites had been reported: U1 sites in papillomaviruses (Furth et al. 1994; Cumming et al. 2003) and artificial U1 sites used to silence expression of reporter and specific endogenous genes (Beckley et al. 2001, Fortes et al. 2003). Prior work from multiple labs showed that these U1 sites use the same mechanism, namely inhibition of pre-mRNA maturation by inhibition of nuclear polyadenylation resulting in lower mRNA levels. This prior work also showed that inhibition requires the $\mathrm{U} 1$ site to be in the $3^{\prime}$ terminal exon and able to form a duplex of 8-10 bp with U1 snRNP (Gunderson et al. 1998; Beckley et al. 2001; Fortes et al. 2003). The U1A gene's U1 site described here has these features.

\section{Synergy and the bipartite element}

The U1A U1 site is found within an unusual bipartite element where the U1 site is repressed by a U1-STEM sequence. Instead of simply blocking U1 snRNP binding, the U1-STEM allows binding but does not permit U1 snRNP to be active unless the nearby PIE is bound by U1A protein. Although the net effect is synergistic, our analysis indicates that it is a property of the ternary complex being more inhibitory to the polyadenylation machinery than each complex individually. This is supported by the in vitro polyadenylation assays in HeLa NE. So why is the ternary complex more inhibitory? The most straightforward explanation is that the U1A:PIE complex releases U1 snRNP from inhibition by the U1-STEM. In this case what we have been calling synergy is, in fact, only an apparent synergy of rescuing U1 snRNP from the U1-STEM repressor rather than a synergy between the inhibitory activities of the U1 and PIE sites. However, an alternative explanation, that a unique, but as yet uncharacterized, feature of the ternary complex has enhanced polyadenylation inhibitory activity, must also be considered. For example, U1A in the U1 snRNP may "jump" over and bind to the PIE site. Although we cannot rule this out, it seems unlikely for several reasons. First, PIE requires two molecules of U1A to inhibit and U1 snRNP has only one U1A molecule. Thus, the "jumping" would only activate a PIE that already had one molecule of bound U1A. Second, it does not explain the high inhibitory activity seen when the U1-STEM and PIE are mutated, as mutation of PIE would prevent such jumping. Furthermore, when U1 snRNP was studied separate from PIE (as in papillomaviruses and HIV-1) we and others showed that the U1-70K subunit of U1 snRNP is necessary and sufficient for polyadenylation inhibition and U1A makes no contribution (Gunderson et al. 1998; Ashe et al. 2000; Beckley et al. 2001; Sajic et al. 2007). Thus, the simpler model is that U1A releases the U1-STEMmediated inhibition of the bound U1 snRNP so that U1$70 \mathrm{~K}$ can now inhibit polyadenylation. However, a thorough understanding of the inhibitory mechanism will require reconstitution of this system with purified factors and a 
better understanding of what is the repressor itself, a topic we now turn to.

\section{Trapping U1 snRNP in a nonproductive complex and parallels with other genes}

An important question is whether U1-STEM-mediated repression requires a trans-acting factor or is repression completely RNA mediated. Such a hypothetical trans-acting factor could be either preassembled in the repressor complex or recruited during the binding of U1 snRNP. A related question is how the U1-STEM traps and holds U1 snRNP if only 4 out of $10 \mathrm{nt}$ of the U1 site are available to base pair to U1 snRNP. Perhaps U1 snRNP base pairs to these $4 \mathrm{nt}$ and then makes additional protein-RNA or RNA-RNA contacts with the U1-STEM or to sequences nearby. Alternatively, the U1-STEM could open to allow U1 snRNP base-pairing to the full U1 site and then additional interactions hold U1 snRNP in an inactive conformation. Additionally, the role of region $\mathrm{C}$ in promoting synergy and why it depends on the U1A-PIE complex will need to be elucidated.

In the context of splicing, the U1 snRNP:5'ss complex is frequently a target of regulation by nearby splicing enhancer or suppressor elements that exert their effect through binding of splicing regulatory factors, most typically members of the SR and hnRNP family (Black 2003). In contrast, examples of regulated splicing based on RNA secondary structure are far less frequent, with most examples involving the branch point and 3 'ss elements rather than the $5^{\prime}$ ss. One $5^{\prime}$ ss-related example that does have similarities with the U1-STEM repressor involves the Saccharomyces cerevisiae L30 protein (formerly called L32). L30 negatively autoregulates its own expression by binding to and inhibiting splicing of a single intron found in its own pre-mRNA (Vilardell and Warner 1994). U1 snRNP is bound to and trapped by an L30 protein:L30 pre-mRNA complex where U1 snRNP base pairs to just half of the 5 'ss (nucleotides 2-6) while the remaining part of the $5^{\prime}$ ss is in a stem structure. The trapping mechanism blocks U1 snRNP from making interactions to carry out the ATPdependent spliceosome assembly steps; consequently, intron removal is blocked, leading to a negative autoregulatory feedback of L30 on its own expression. However, whether L30 or the RNA:5'ss stem or both directly repress U1 snRNP and what part of U1 snRNP is repressed has remained unresolved. Another example of a trapped U1 snRNP is the Drosophila P-element Somatic Inhibitor (PSI) protein, which, in somatic cells, inhibits splicing of the $\mathrm{P}$ element intron 3 by tethering U1 snRNP to a decoy $5^{\prime}$ ss sequence near the authentic $5^{\prime}$ ss (Labourier et al. 2001). Interestingly, PSI tethers and inactivates $\mathrm{U} 1$ snRNP by binding to U1-70K, a U1 snRNP subunit that makes numerous key interactions with splicing regulatory proteins (Black 2003) and is also responsible for inhibition of polyadenylation, as it contains four polyadenylation regulatory domains (PRDs) that directly bind to and inhibit polyA polymerase (Gunderson et al. 1998). If one uses the PSI analogy, the U1 snRNP trapping mechanism could plausibly involve the PRDs of U1-70K being masked by the U1-STEM or a hypothetical polypeptide that binds at or near the U1-STEM. Current studies are underway to test these possibilities.

\section{Prevalence and regulation of $\mathrm{U} 1$ sites}

As this is the first example of a $3^{\prime}$ terminal U1 site in a cellular gene it is only natural to wonder how many more there are. Our 3'UTR database search yielded other genes with conserved U1 sites that we are now investigating. Whether these U1 sites are autonomous or depend on nearby elements will require a combination of bioinformatics, sequence conservation, and testing candidate $\mathrm{U} 1$ sites in reporter genes. We caution that such testing would have initially given misleading information in the case of the U1A U1 site, since its activity was repressed by the U1-STEM. Candidate U1 sites deemed to be inactive by reporter gene assays will have to be scrutinzed for potential base-pairing interactions with flanking elements. In the case of papillomavirus U1 sites, it is evident that a differentiationdependent repression of the $\mathrm{U} 1$ site must occur in keratinocytes in order to release the viral polyA site for high expression of the viral late genes necessary to make virions. The difficulty in recapitulating this release in cultured cells has hindered elucidation of the mechanism.

Internal PAS that occur in introns are a second class of U1 site-containing genes where the upstream unused $5^{\prime}$ ss can, in fact, be a potential U1 site able to inhibit the internal PAS. Recent bioinformatic analysis estimates that $20 \%$ of human genes have such internal polyA sites (Tian et al. 2007). The IgM gene represents a well-characterized example of a regulated internal PAS, the secretory PAS, and indeed, mutation of its unused $5^{\prime}$ ss leads to increased expression in reporter genes containing the secretory PAS region. As this same PAS is also regulated by U1A binding sites (Phillips et al. 2001, 2004), it will be of interest to determine how they interact with the upstream U1 snRNP:5'ss complex.

\section{Significance for autoregulation of U1A}

The question remains: Why does the U1A gene have such a complex collection of inhibitory elements? One possibility, that the bipartite U1 site is acting to amplify the negative autoregulatory feeback of the U1A:PIE complex, is not consistent with our previous finding that the U1A autoregulatory system could be made stronger by simple amino acid substitutions in the U1A protein (Guan et al. 2003). Another possibility is that it may be important to integrate regulation of U1 snRNP and U1A levels. A third possibility 
hinges on whether a trans-acting factor is found to bind and affect the bipartite element. This factor could, in principle, allow other proteins or pathways to regulate U1A levels leading to downstream effects on expression levels of genes whose polyA sites are regulated by U1A.

\section{MATERIALS AND METHODS}

\section{Oligo treatment of NE}

Anti-U1 oligo-treated NE and Northern and Western blotting were done as previously described (Gunderson et al. 1998). The anti-U1A antibody 856 (kindly provided by Iain Mattaj, EMBL, Heidelberg, Germany) and anti-Flag antibody (Sigma) were diluted 1:5,000; the anti-GAPDH antibody (Chemicon) was diluted 1:30,000.

\section{Biotin pull-down assay}

Streptavidin beads (Sigma) were washed three times in $1-\mathrm{mL}$ binding buffer (BB, $150 \mathrm{mM} \mathrm{NaCl}, 20 \mathrm{mM}$ HEPES pH 7.9, 0.03\% $\mathrm{NP}-40,2 \mathrm{mM} \mathrm{MgCl}_{2}$ ) and then preblocked in $700 \mu \mathrm{L} \mathrm{BB}, 100 \mu \mathrm{g}$ BSA, $50 \mu \mathrm{g}$ tRNA, and $100 \mu \mathrm{g}$ glycogen by rotating for $30 \mathrm{~min}$. After three more washes the beads were split to each reaction tube (10 $\mu \mathrm{L} /$ tube) and resuspended in $100 \mu \mathrm{L} \mathrm{BB}, 96 \mu \mathrm{mol}$ DTT, $10 \mu \mathrm{g}$ tRNA, 40 units RNasin, and an RNA probe. After 30 min of rotation the beads were washed two times and resuspended in $72 \mu \mathrm{L} \mathrm{BB}, 96 \mu \mathrm{mol}$ DTT, $12 \mu \mathrm{L}$ 90\% glycerol, $10 \mu \mathrm{g}$ tRNA, 40 units of RNasin, and varying amounts of HeLa NE and rU1A. After $30 \mathrm{~min}$ of rotation the beads were washed three times with $750 \mu \mathrm{L}$ $\mathrm{BB}$ with $237 \mathrm{mM} \mathrm{NaCl}$. Specifically bound RNA and proteins were eluted by addition of Laemmli buffer, and half the eluate analyzed by Western blotting and the other half analyzed by Northern blotting.

\section{Transfections}

HeLaTet cells, expressing the reverse Tetracycline Repressor (Clontech), and HeLa cells were passaged in standard condition and transfected with Polyfect (Qiagen) as per manufacturer's instructions. Each FLAG-tagged U1A expression construct (100 ng) (Figs. 2,3) were transfected into 1.5 million HelaTet cells on a 6-cm plate along with $100 \mathrm{ng}$ of Flag-PRP28, that serves as a transfection efficiency control. Forty-eight hours after transfection, cells were harvested and lysed in SDS-Laemmli buffer for Western blotting. For the Renilla assays in Figures 2 and 3, 10 ng of each Renilla reporter were transfected into 150,000 HeLaTet cells on a 24 -well plate along with $10 \mathrm{ng}$ of a Firefly luciferase plasmid that controls transfection efficiency. Forty-eight hours after transfection luciferase activity was measured using the dual luciferase assay kit (Promega) as per manufacturer's instructions.

\section{Western blotting}

Samples were separated onto a 12\% SDS-PAGE gel. Afterward, the gel was taken down and transferred to an Immobilon-P membrane (Millipore) in transfer buffer (192 mM Glycine, $25 \mathrm{mM}$ Tris, $20 \% \mathrm{v} / \mathrm{v}$ methanol) at $300 \mathrm{~mA}, 25 \mathrm{~V}$, and $10 \mathrm{~W}$ for $12 \mathrm{~h}$. The membrane was then blocked with $1 \times$ PBS, $0.1 \%$ Triton, $0.7 \%-5 \% \mathrm{w} / \mathrm{v}$ milk powder for $30-60 \mathrm{~min}$. The membrane then was probed with the primary antibody in new blocking solution. For the anti-U1A antibody 856 (Kambach and Mattaj 1992) or anti-Flag antibody (Sigma), a dilution of 1:5,000 was generally employed while the anti-GAPDH antibody (Chemicon) was diluted 1:30,000. After 1-2 h of rocking, the membrane was washed twice with $1 \times$ PBS $+0.1 \%$ Triton, 5 min for each wash. Then the membrane was incubated with the corresponding antispecies-specific Horseradish peroxidase (HRP) antibody (Amersham) for $1 \mathrm{~h}$. After being washed twice with $1 \times \mathrm{PBS}+0.1 \%$ Triton $+1 \%$ milk powder, twice with $1 \times \mathrm{PBS}+0.1 \%$ Triton, and once with $1 \times$ PBS, 5 min each time, the membrane was soaked in ECL reagent (Perkin-Elmer) for $60 \mathrm{sec}$ and exposed to X-ray film.

\section{Northern blotting}

RNA samples were first separated on an $8 \%$ (24:1) ureaacrylamide denaturing gel. The gel was then transferred to an Hybond N+ membrane (Amersham) in TBE buffer (45 mM Tris, $45 \mathrm{mM}$ boric acid, $1.25 \mathrm{mM}$ EDTA, pH 8.3) at $300 \mathrm{~mA}, 10 \mathrm{~V}, 5 \mathrm{~W}$ for $3 \mathrm{~h}$. After one wash in $\mathrm{ddH}_{2} \mathrm{O}$, the wet membrane was UVcross-linked twice in a Stratalinker, $30 \mathrm{sec}$ each time. The membrane was then air dried and prehybridized at $42^{\circ} \mathrm{C}$ by rocking for $30 \mathrm{~min}$ in prehyb buffer consisting of $50 \% \mathrm{v} / \mathrm{v}$ formamide, $5 \times$ SSC buffer [0.75M NaCl, $75 \mathrm{mM}$ sodium citrate, $\mathrm{pH} 7.0], 5 \times$ Denhardts buffer [ $1 \mathrm{mg} / \mathrm{mL}$ BSA, $1 \mathrm{mg} / \mathrm{mL}$ PVP polypropyl, 1 $\mathrm{mg} / \mathrm{mL}$ Ficoll-400], $1 \%$ SDS, and $0.1 \mathrm{mg} / \mathrm{mL}$ sonicated salmon sperm DNA. Ten million dpm of 32P-RNA probes, either anti-U1 snRNA alone or with anti-U2 snRNA, were then added to the prehyb buffer and incubated with the membrane at $42^{\circ} \mathrm{C}$ by rocking for $12 \mathrm{~h}$ or overnight. After hybridization, the membrane was washed twice with $2 \times$ SSC buffer $(0.3 \mathrm{M} \mathrm{NaCl}, 30 \mathrm{mM}$ sodium citrate), 5 min each time at room temperature; twice with $2 \times$ SSC buffer, $0.1 \%$ SDS, 30 min each time at $55^{\circ} \mathrm{C}-60^{\circ} \mathrm{C}$; and twice with $2 \times$ SSC, 1 min each time at room temperature. Finally the membrane was exposed to X-ray film.

\section{In vitro polyadenylation assays}

In vitro polyadenylation assays were performed as described (Boelens et al. 1993; Gunderson et al. 1994). Assays that included $\mathrm{U} 1$ oligo treated NE were done as described in Gunderson et al. (1998).

\section{SUPPLEMENTAL DATA}

Supplemental material on the U1A protein preparations used in this work can be obtained by e-mailing gunderson@biology. rutgers.edu.

\section{ACKNOWLEDGMENTS}

This work was supported by NIH RO1 grant GM057286 to S.I.G., NJCCR Grant 05-2004-CCR-EO to R.G., and a fellowship from Rutgers IGERT program on Biointerfaces to E.S.H. from NSF DGE 0333196 (PI: P. Moghe). We thank members of the Gunderson laboratory and the Rutgers/UMDNJ RNA community for advice and comments during the course of this work.

Received July 25, 2007; accepted August 27, 2007. 


\section{REFERENCES}

Arhin, G.K., Boots, M., Bagga, P.S., Milcarek, C., and Wilusz, J. 2002. Downstream sequence elements with different affinities for the hnRNP $\mathrm{H} / \mathrm{H}^{\prime}$ protein influence the processing efficiency of mammalian polyadenylation signals. Nucleic Acids Res. 30: 18421850. doi: 10.1093/nar/30.8.1842.

Ashe, M.P., Furger, A., and Proudfoot, N.J. 2000. Stem-loop 1 of the U1 snRNP plays a critical role in the suppression of HIV-1 polyadenylation. RNA 6: 170-177.

Beaudoing, E. and Gautheret, D. 2001. Identification of alternate polyadenylation sites and analysis of their tissue distribution using EST data. Genome Res. 11: 1520-1526.

Beckley, S.A., Liu, P., Stover, M.L., Gunderson, S.I., Lichtler, A.C., and Rowe, D.W. 2001. Reduction of target gene expression by a modified U1 snRNA. Mol. Cell. Biol. 21: 2815-2825.

Black, D.L. 2003. Mechanisms of alternative pre-messenger RNA splicing. Annu. Rev. Biochem. 72: 291-336.

Boelens, W.C., Jansen, E.J.R., van Venrooij, W.J., Stripecke, R., Mattaj, I.W., and Gunderson, S.I. 1993. The human U1 snRNPspecific U1A protein inhibits polyadenylation of its own premRNA. Cell 72: 881-892.

Cumming, S.A., McPhillips, M.G., Veerapraditsin, T., Milligan, S.G., and Graham, S.V. 2003. Activity of the human papillomavirus type 16 late negative regulatory element is partly due to four weak consensus $5^{\prime}$ splice sites that bind a U1 snRNP-like complex. J. Virol. 77: 5167-5177.

Edmonds, M. 2002. A history of poly A sequences: From formation to factors to function. Prog. Nucleic Acid Res. Mol. Biol. 71: 285-389.

Fortes, P., Cuevas, Y., Guan, F., Liu, P., Pentlicky, S., Jung, S.P., Chantar, M.L., Prieto, J., Rowe, D., and Gunderson, S.I. 2003. Inhibiting expression of specific genes in mammalian cells using modified U1 snRNPs targeted to terminal exons of pre-mRNA. Proc. Natl. Acad. Sci. 100: 8264-8269.

Furth, P.A., Choe, W., Rex, J.H., Byrne, J.C., and Baker, C.C. 1994. Sequences homologous to $5^{\prime}$ splice sites are required for the inhibitory activity of papillomavirus late $3^{\prime}$ untranslated regions. Mol. Cell. Biol. 14: 5278-5289.

Guan, F., Palacios, D., Hussein, R.I., and Gunderson, S.I. 2003. Determinants within an 18-amino-acid U1A autoregulatory domain that uncouple cooperative RNA binding, inhibition of polyadenylation, and homodimerization. Mol. Cell. Biol. 23: 3163-3172.

Guhaniyogi, J. and Brewer, G. 2001. Regulation of mRNA stability in mammalian cells. Gene 265: 11-23.

Gunderson, S.I., Beyer, K., Martin, G., Keller, W., Boelens, W.C., and Mattaj, I.W. 1994. The human U1A snRNP protein regulates polyadenylation via a direct interaction with poly(A) polymerase. Cell 76: 531-541.

Gunderson, S.I., Vagner, S., Polycarpou-Schwarz, M., and Mattaj, I.W. 1997. Involvement of the carboxyl terminus of vertebrate poly(A) polymerase in U1A autoregulation and in the coupling of splicing and polyadenylation. Genes \& Dev. 11: 761-773.

Gunderson, S.I., Polycarpou-Schwarz, M., and Mattaj, I.W. 1998. U1 snRNP inhibits pre-mRNA polyadenylation through a direct interaction between U170K and poly(A) polymerase. Mol. Cell 1: 255-264.

Kambach, C. and Mattaj, I.W. 1992. Intracellular distribution of the U1A protein depends on active transport and nuclear binding to U1 snRNA. J. Cell Biol. 118: 11-21.

Krämer, A. and Keller, W. 1990. Preparation and fractionation of mammalian extracts active in pre-mRNA splicing. Methods Enzymol. 181: 3-19.

Labourier, E., Adams, M.D., and Rio, D.C. 2001. Modulation of Pelement pre-mRNA splicing by a direct interaction between PSI and U1 snRNP 70K protein. Mol. Cell 8: 363-373.
Lou, H., Neugebauer, K.M., Gagel, R.F., and Berget, S.M. 1998. Regulation of alternative polyadenylation by U1 snRNPs and SRp20. Mol. Cell. Biol. 18: 4977-4985.

Ma, J., Gunderson, S.I., and Phillips, C. 2006. Non-snRNP U1A levels decrease during mammalian $\mathrm{B}$-cell differentiation and release the IgM secretory poly(A) site from repression. RNA 12: 122-132.

Milcarek, C., Martincic, K., Chung-Ganster, L.H., and Lutz, C.S. 2003. The snRNP-associated U1A levels change following IL-6 stimulation of human B-cells. Mol. Immunol. 39: 809-814.

Pesole, G., Liuni, S., Grillo, G., Licciulli, F., Mignone, F., Gissi, C., and Saccone, C. 2002. UTRdb and UTRsite: Specialized databases of sequences and functional elements of $5^{\prime}$ and $3^{\prime}$ untranslated regions of eukaryotic mRNAs. Nucleic Acids Res. 30: 335-340. doi: $10.1093 / \mathrm{nar} / 30.1 .335$

Pettitt, J., Crombie, C., Schumperli, D., and Muller, B. 2002. The Caenorhabditis elegans histone hairpin-binding protein is required for core histone gene expression and is essential for embryonic and postembryonic cell division. J. Cell Sci. 115: 857-866.

Phillips, C., Jung, S., and Gunderson, S.I. 2001. Regulation of nuclear poly(A) addition controls the expression of immunoglobulin $\mathrm{M}$ secretory mRNA. EMBO J. 20: 6443-6452.

Phillips, C., Pachikara, N., and Gunderson, S.I. 2004. U1A inhibits cleavage at the immunoglobulin $\mathrm{M}$ heavy-chain secretory poly(A) site by binding between the two downstream GU-rich regions. Mol. Cell. Biol. 24: 6162-6171.

Sajic, R., Lee, K., Asai, K., Sakac, D., Branch, D.R., Upton, C., and Cochrane, A. 2007. Use of modified U1 snRNAs to inhibit HIV-1 replication. Nucleic Acids Res. 35: 247-255. doi: 10.1093/nar/gkl1022.

Shell, S.A., Hesse, C., Morris Jr., S.M., and Milcarek, C. 2005. Elevated levels of the $64-\mathrm{kDa}$ cleavage stimulatory factor (CstF-64) in lipopolysaccharide-stimulated macrophages influence gene expression and induce alternative poly(A) site selection. J. Biol. Chem. 280: 39950-39961.

Takagaki, Y., Seipelt, R.L., Peterson, M.L., and Manley, J.L. 1996. The polyadenylation factor CstF-64 regulates alternative processing of IgM heavy chain pre-mRNA during B-cell differentiation. Cell 87: 941-952.

Thompson, J.D., Higgins, D.G., and Gibson, T.J. 1994. CLUSTAL W: Improving the sensitivity of progressive multiple sequence alignment through sequence weighting, position-specific gap penalties and weight matrix choice. Nucl. Acids Res. 22: 4673-4680. doi: 10.1093/ nar/22.22.4673

Tian, B., Hu, J., Zhang, H., and Lutz, C.S. 2005. A large-scale analysis of mRNA polyadenylation of human and mouse genes. Nucleic Acids Res. 33: 201-212. doi: 10.1093/nar/gki158.

Tian, B., Pan, Z., and Lee, J.Y. 2007. Widespread mRNA polyadenylation events in introns indicate dynamic interplay between polyadenylation and splicing. Genome Res. 17: 156-165.

Venkataraman, K., Brown, K.M., and Gilmartin, G.M. 2005. Analysis of a noncanonical poly(A) site reveals a tripartite mechanism for vertebrate poly(A) site recognition. Genes \& Dev. 19: 1315-1327.

Vilardell, J. and Warner, J.R. 1994. Regulation of splicing at an intermediate step in the formation of the spliceosome. Genes \& Dev. 8: 211-220.

Will, C.L. and Lührmann, R. 1997. Protein functions in pre-mRNA splicing. Curr. Opin. Cell Biol. 9: 320-328.

Wilusz, C.J., Wormington, M., and Peltz, S.W. 2001. The cap-to-tail guide to mRNA turnover. Nat. Rev. Mol. Cell Biol. 2: 237-246.

Yan, J. and Marr, T.G. 2005. Computational analysis of $3^{\prime}$-ends of ESTs shows four classes of alternative polyadenylation in human, mouse, and rat. Genome Res. 15: 369-375.

Zhao, J., Hyman, L., and Moore, C. 1999. Formation of mRNA 3' ends in eukaryotes: Mechanism, regulation, and interrelationships with other steps in mRNA synthesis. Microbiol. Mol. Biol. Rev. 63: 405-445. 

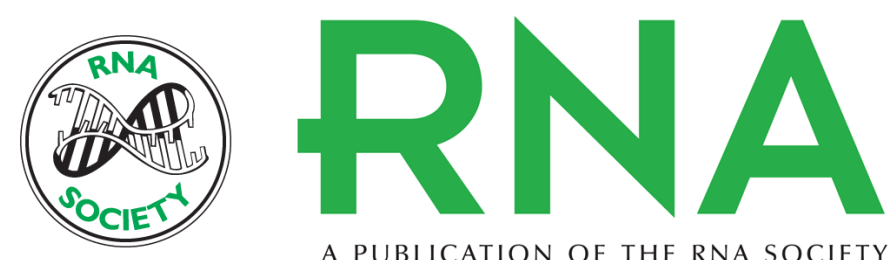

A PUBLICATION OF THE RNA SOCIETY

\section{A bipartite U1 site represses U1A expression by synergizing with PIE to inhibit nuclear polyadenylation}

Fei Guan, Rose M. Caratozzolo, Rafal Goraczniak, et al.

RNA 2007 13: 2129-2140

References This article cites 37 articles, 20 of which can be accessed free at:

http://rnajournal.cshlp.org/content/13/12/2129.full.html\#ref-list-1

License

Email Alerting Receive free email alerts when new articles cite this article - sign up in the box at the Service top right corner of the article or click here. 